\title{
Effective Neutrino Mass Operators and Their Applications
}

\author{
C.N. Leung* ${ }^{\dagger}$ \\ Department of Physics and Astronomy \\ University of Delaware, Newark, DE 19716, U.S.A. \\ E-mail: 'leung@physics.udel.edü
}

ABSTRACT: We consider effective operators relevant for generating small Majorana masses for the neutrinos in the Standard Model and show how they can be used to systematically construct neutrino mass models. Some of these operators reproduce well-known models in the literature, others lead to interesting new models. It is shown that neutrinoless double beta decay experiments will potentially be very helpful for identifying the most relevant operators.

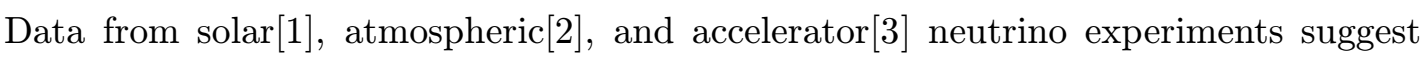
that neutrinos have small masses of order $1 \mathrm{eV}$ or less. While it is possible to accommodate nonzero neutrino masses by modifying the low-energy particle content of the Standard Model (e.g., by introducing an $S U(2)_{L}$ triplet scalar field which acquires a vacuum

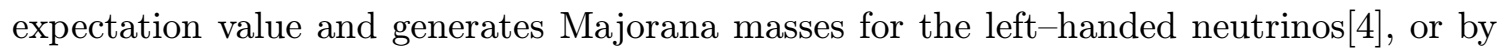
introducing right-handed neutrinos so that Dirac neutrino masses can be generated), the smallness of the neutrino masses relative to the masses of the quarks and charged leptons remain a puzzle in such attempts.

Why the masses of the neutrinos are so small can be understood in a natural way if they are generated via some underlying new physics characterized by an energy scale $\Lambda$ which is much higher than the electroweak scale. Typically, $\Lambda$ corresponds to the scale at which the lepton number symmetry is broken. In this case, neutrino masses will be described at low energies (i.e., at or below the electroweak scale) by higher dimensional $(d>4)$ effective operators which are suppressed by appropriate powers of $\Lambda$, and their smallness can be understood from purely dimensional grounds.

A familiar example of this effective-operator description is the seesaw mechanism in which heavy $S U(2)_{L}$ singlet right-handed neutrino fields, $N_{1}$, are introduced. The $N_{1}$ fields can form a Dirac mass term with the left-handed neutrinos. Upon integrating out

${ }^{*}$ Speaker.

${ }^{\dagger}$ This work was supported in part by the U.S. Department of Energy under grant DE-FG02-84ER40163. 
the heavy $N_{1}$ fields, one obtains an effective theory without the right-handed neutrinos,

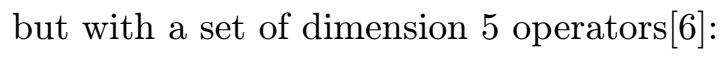

$$
\mathcal{O}_{1}=\frac{g_{a b}^{(1)}}{\Lambda}\left(L_{a}^{i T} C L_{b}^{j}\right) H^{k} H^{l} \epsilon_{i k} \epsilon_{j l}
$$

where $L$ stands for the left-handed lepton doublets, $H$ is the Standard Model Higgs doublet, $C$ denotes the charge conjugation operator, $\{i, j, k, \ldots\}$ are $S U(2)_{L}$ indices, the subscripts $\{a, b, \ldots\}$ label fermion generations, and $g_{a b}^{(1)}$ are dimensionless coefficients which are determined by the underlying new physics (e.g., an $S O(10)$ grand unified theory). When the neutral component of $H$ develops a vacuum expectation value, $v$, the operators in $\mathcal{O}_{1}$ will generate Majorana neutrino masses of order $v^{2} / \Lambda$. This is illustrated in Fig. 1 of Ref. [i]i], where it is pointed out that, instead of $N_{1}, \mathcal{O}_{1}$ can also be induced by fermions $\left(N_{3}\right)$ or

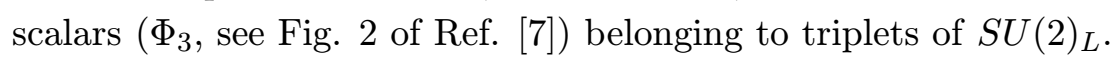

In order for $\mathcal{O}_{1}$ to generate neutrino masses of order $1 \mathrm{eV}$ or less, $\Lambda$ must be greater than $10^{13} \mathrm{GeV}$ or so. On the other hand, limits from lepton number violating processes such as $\mu \rightarrow e e e$ and $\mu \rightarrow e \gamma$ only constrain $\Lambda$ to be larger than a few TeV. If the actual lepton number breaking scale is closer to this experimental lower bound ${ }^{1}, \mathcal{O}_{1}$ will generate too large a neutrino mass and other effective neutrino mass operators will have to be considered. In view of the current interests in neutrino mass models, it will be useful to

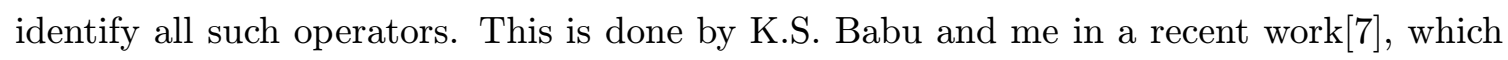
is the subject of my report here.

Since we are interested in effective operators that can induce Majorana masses for the left-handed neutrinos in the Standard Model, the operators under consideration must violate lepton number by two units. They must be $S U(3)_{C} \times S U(2)_{L} \times U(1)_{Y}$ invariant and contain only fields that are present in the Standard Model Lagrangian ${ }^{2}$. In addition, the operators are required to conserve baryon number, otherwise the nonobservation of proton decays will limit $\Lambda$ to be larger than $10^{14} \mathrm{GeV}$ and the resulting neutrino masses will be too small to be of interest.

The full list of effective neutrino mass operators can be found in Ref. [i] grouped according to the number of fermion fields they contain. Operators containing two fermion fields include only the dimension five seesaw operators, which, as discussed above,

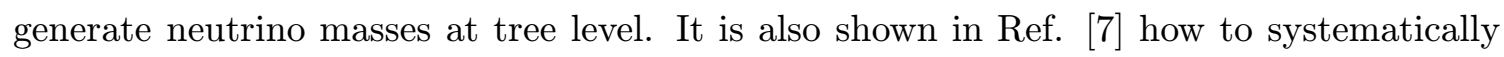
construct renormalizable neutrino mass models from the effective operators. As an example, we saw earlier that $\mathcal{O}_{1}$ might arise from models containing heavy $N_{1}, N_{3}$, or $\Phi_{3}$ fields.

While the $d=5$ seesaw operators are the lowest dimensional effective neutrino mass operators, there may be situations in which they are not the suitable ones. For instance, as exemplified in many of the models discussed in Ref. [i]. there may be selection rules in the underlying theory which forbid their presence. Such selection rules may be necessary

\footnotetext{
${ }^{1}$ This may be the case if lepton number is broken by quantum effects of gravity. Then $\Lambda$ will be of order the Planck scale which can be as low as a few $\mathrm{TeV}$ if large extra dimensions exist.

${ }^{2}$ In addition to $L$ and $H$ in $\mathcal{O}_{1}$, there are the left-handed quark doublets, $Q$, and the charge conjugates of the right-handed charged leptons, up-type quarks and down-type quarks: $e^{c}=C{\overline{e_{R}}}^{T}, u^{c}$ and $d^{c}$.
} 
for models in which the lepton number breaking scale $\Lambda$ is sufficiently low that $\mathcal{O}_{1}$ would generate too large a neutrino mass (larger than $\mathrm{O}(1) \mathrm{eV}$ ). In such cases, the dominant contributions to the neutrino masses will come from operators with dimensions higher than 5 , typically through radiative corrections.

Operators containing four fermion fields generate neutrino masses radiatively at the one-loop level. A well studied model of one-loop radiative neutrino mass generation is the Zee model[ [8], which finds its low-energy realization in the dimension seven operator

$$
\mathcal{O}_{2}=\frac{g^{(2)}}{\Lambda^{3}} L^{i} L^{j} L^{k} e^{c} H^{l} \epsilon_{i j} \epsilon_{k l}
$$

where the generation indices and Lorentz contractions on the fermion fields have been suppressed. Similarly, the operator, $\mathcal{O}_{3}=\frac{g^{(3)}}{\Lambda^{3}} L^{i} L^{j} Q^{k} d^{c} H^{l} \epsilon_{i k} \epsilon_{j l}$, is realized in the supersymmetric standard model with $R$-parity violation [ $[9]$. As an example of a new neutrino mass model, consider the operator

$$
\mathcal{O}_{4}=\frac{g^{(4)}}{\Lambda^{3}} L^{i} L^{j} \bar{Q}_{i} \bar{u}^{c} H^{k} \epsilon_{j k}
$$

which can be induced through the renormalizable Lagrangian:

$$
\mathcal{L}^{\mathcal{O}_{4}}=\bar{Q}_{i} \gamma_{\mu} L^{i} G_{1}^{\mu}+\bar{u}^{c} \gamma_{\mu} L^{i} G_{2 i}^{\mu *}+G_{1}^{\mu *} G_{2 \mu}^{i} H^{j} \epsilon_{i j}+\text { h.c. }
$$

where $G_{1}^{\mu}$ is an $S U(2)_{L}$ singlet leptoquark gauge boson, $G_{2}^{\mu}$ is a leptoquark gauge boson that is an $S U(2)_{L}$ doublet, and the coupling constant for each interaction term is not shown explicitly. This model can be embedded into an $S O(10)$ grand unified model [i]j].

Operators containing six fermion fields typically generate neutrino masses via two-loop radiative corrections. Because of the additional suppression factors, the scale $\Lambda$ in this class of models are generally much lower than the seesaw scale, and may even be close to the electroweak scale. An example of this class of operators is

$$
\mathcal{O}_{11}=\frac{g^{(11)}}{\Lambda^{5}} L^{i} L^{j} Q^{k} Q^{l} d^{c} d^{c} \epsilon_{i k} \epsilon_{j l}
$$

which may arise from the renormalizable interactions

$$
\mathcal{L}^{\mathcal{O}_{11}}=f_{a b} L_{a} L_{b} \Phi_{1}+g_{a b} Q_{a} Q_{b} \Omega_{1}+h_{a b} d_{a}^{c} d_{b}^{c} \bar{\Omega}_{1}+\mu \Phi_{1} \bar{\Omega}_{1} \Omega_{1}+\text { h.c. }
$$

Here $\Phi_{1}$ is an $S U(3)_{C}$ and $S U(2)_{L}$ singlet scalar field, and $\Omega_{1}\left(\bar{\Omega}_{1}\right)$ are scalar fields transforming as a triplet (anti-triplet) under $S U(3)_{C}$ and a singlet under $S U(2)_{L}$.

We have not considered operators with eight or more fermion fields because operators with $d \geq 12$ will be highly suppressed and will generate neutrino masses that are too small to satisfy the atmospheric neutrino data which require at least one neutrino to have a mass of about $0.03 \mathrm{eV}$. Even with this truncation, our list of effective neutrino mass operators contains a large number of operators. It will be helpful if the number of relevant operators may be reduced. To this end, neutrinoless double beta $(0 \nu 2 \beta)$ decays may show the way. This is because there is a subset of operators (e.g., $\mathcal{O}_{11}, L^{i} d^{c} \bar{Q}_{j} \bar{u}^{c} \bar{e}^{c} \bar{u}^{c} \bar{H}_{i} H^{j}$, 
etc.) which can produce $0 \nu 2 \beta$ decays directly, but can only generate neutrino masses at the two-loop level. These operators offer the interesting possibility that the $0 \nu 2 \beta$ decay amplitudes they generate may be large enough to be observable in current experiments even though the neutrino masses they induce are small enough to be consistent with the solar and atmospheric neutrino data. Thus, if $0 \nu 2 \beta$ decays are observed in the current round of experiments, this subset of operators will be singled out as the most relevant effective neutrino mass operators.

\section{References}

[1] B.T. Cleveland et al., Nucl. Phys. B (Proc. Suppl.) 38 (1995) 47; K.S. Hirata et al., Phys. Rev. D 44 (1991) 2241; GALLEX Collaboration, W. Hampel et al., Phys. Lett. B388 (1996) 384; SAGE Collaboration, J.N. Abdurashitov et al., Phys. Rev. Lett. 77 (1996) 4708.

[2] Super-Kamiokande Collaboration, Y. Fukuda et al., Phys. Lett. B433 (1998) 9; Phys. Lett. B436 (1998) 33; Phys. Rev. Lett. 81 (1998) 1562.

[3] LSND Collaboration, C. Athanassopoulos et al., Phys. Rev. Lett. 75 (1995) 2650; W.C. Louis for the LSND Collaboration, in Particle Physics and Cosmology, ed. J. Nieves (Amer. Inst. Phys., New York, 2000), p. 93.

[4] T.P. Cheng and L.F. Li, Phys. Rev. D 22 (1980) 2860.

[5] M. Gell-Mann, P. Ramond and R. Slansky, in Supergravity, eds. P. van Nieuwenhuizen and D. Freedman (North Holland, Amsterdam, 1979), p. 315; T. Yanagida, in Workshop on the Unified Theory and Baryon Number in the Universe, eds. O. Sawada and A. Sugamoto (KEK, Tsukuba, 1979), p. 95.

[6] S. Weinberg, Phys. Rev. Lett. 43 (1979) 1566.

[7] K.S. Babu and C.N. Leung, hep-ph/0106054, Nucl. Phys. B (in press).

[8] A. Zee, Phys. Lett. B 93 (1980) 389; Erratum - ibid. 95 (1980) 461.

[9] L. Hall and M. Suzuki, Nucl. Phys. B 231 (1984) 419. 